\title{
Serum Biochemistry in Four Siberian Husky Puppies with Swimming Puppy Syndrome
}

\author{
Korakot Nganvongpanit ${ }^{1,2}$ \\ ${ }^{1}$ Bone and Joint Research Laboratory, Department of Veterinary Biosciences and Public Health, Faculty of Veterinary Medicine, \\ Chiang Mai University, Chiang Mai, Thailand \\ ${ }^{2}$ Materials Science Research Center, Faculty of Science, Chiang Mai University, Chiang Mai, Thailand \\ Email: korakot.n@cmu.ac.th
}

Received September 14, 2012; revised October 20, 2012; accepted October 27, 2012

\begin{abstract}
Complete blood counts and blood chemistry in four Siberian husky puppies diagnosed with swimming puppy syndrome were compared with four healthy Siberian husky puppies. Only serum creatine kinase in affected puppies was significantly $(P<0.01)$ higher than in normal puppies. Although serum creatine kinase level cannot be used for diagnosis of this disease, it could serve as a tool for prognosis of the disease after treatment.
\end{abstract}

Keywords: Swimming Puppy Syndrome; Serum Biochemistry; Creatine Kinase

\section{Introduction}

Swimming puppy syndrome is one of the mystery diseases in dogs. Many of the physiological changes that occur in the body are still unclear, and the cause of the disease is uncertain as well. All affected puppies show hyperextention of the stifle and hock joints as well as abduction of the hip joint. In some puppies forelimb lesions are present, including abduction of the shoulder joint, extension of the elbow joint and flexion of the carpal joint. Some puppies show pectus excavatum, which has a poor prognosis. Although many publications have reported that cases are rare in small animal clinics [1], our previous study found that $2 \%$ of puppies were affected (data in process of publication). Due to the limited number of published reports on swimming puppy syndrome, there is a poor understanding of the precise mechanism of disease. At the present time, no studies have been published on the serum biochemistry in puppies affected with swimming puppy syndrome. This case report is the first to include complete blood count and blood chemistry in these animals. This information will help scientists understand the changes of body structure in puppies affected with swimming puppy syndrome. The present study analyzed the serum biochemistry in four Siberian husky puppies affected with swimming puppy syndrome and compared the results with normal values and with blood samples from four healthy Siberian husky puppies in the same age range.

\section{Case Description}

Four Siberian husky puppies were diagnosed with swimming puppy syndrome based on clinical signs (Figure 1). Three puppies were affected in both hind limbs, while the other was affected in both fore and hind limbs.

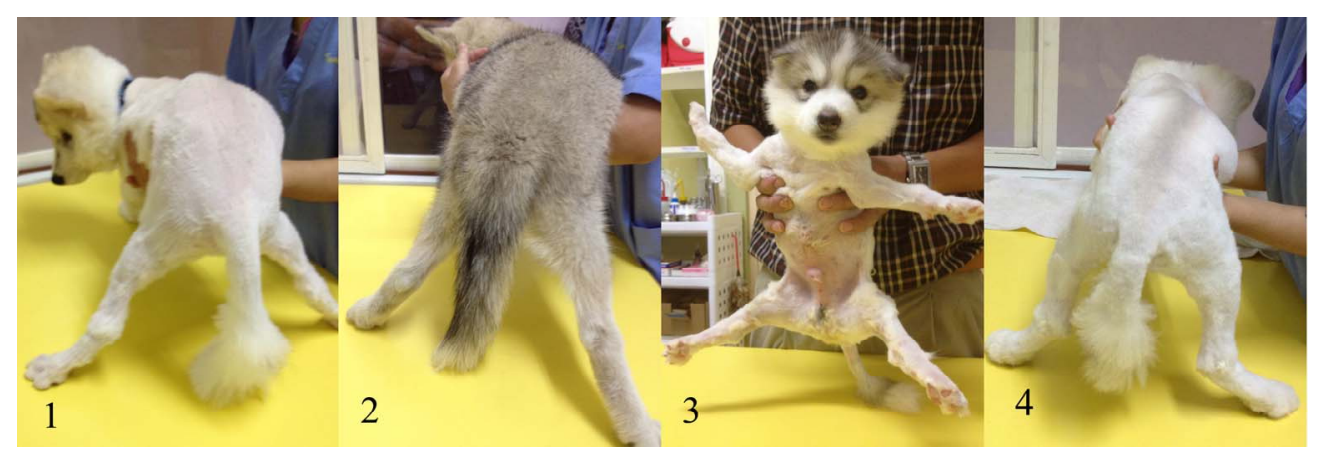

Figure 1. Four Siberian husky puppies affected with swimming puppy syndrome; all puppies, aged 6 - 8 weeks, show extension of the stifle joint and abduction of the hip joint. 
Patient information is presented in Table 1. Another four healthy Siberian husky puppies served as controls (Table 2). Blood samples were collected twice: on the animal's first visit to the hospital, and again 1 month later. By the second visit, all dogs with swimming puppy syndrome showed improvement of clinical signs, and were able to walk on all four legs. All affected puppies were treated by the same method: 1 week realignment of the affected limb, followed by rehabilitation including standing training, standing on a balance board, and swimming every day. During treatment the puppies did not receive any medicine.

Blood was collected from the cephalic vein of each puppy and a complete blood count analysis was performed, including hematocrit and hemoglobin levels, red blood cell count, white blood cell count and platelet count. Serum samples were analyzed for aspartate aminotransferase (ALP), alanine aminotransferase (ALT), alkaline phosphatase (ALP), blood urea nitrogen (BUN), creatine kinase $(\mathrm{CK})$ and creatinine. All blood evaluations were performed at a private veterinary diagnostic laboratory.

Each variable was presented as mean and standard derivation. A paired $t$-test was used to compare differences between affected and healthy groups; significance was set at $P<0.05$.
Table 1. Patient puppy information.

\begin{tabular}{ccccc}
\hline \multirow{2}{*}{ Parameter } & \multicolumn{4}{c}{ Puppy affected with swimming puppy syndrome } \\
\cline { 2 - 5 } & 1 & 2 & 3 & 4 \\
\hline Sex & Male & Male & Male & Female \\
Age (weeks) & 8 & 6 & 7 & 8 \\
Weight (kg) & 2.6 & 2.5 & 1.6 & 2.8 \\
$\begin{array}{c}\text { Limb } \\
\text { affected }\end{array}$ & hind limb & hind limb & fore/hind limb & hind limb \\
$\begin{array}{c}\text { Number of } \\
\text { puppies/litter }\end{array}$ & 4 & 2 & 1 & 4 \\
\hline
\end{tabular}

Table 2. Control puppy information.

\begin{tabular}{ccccc}
\hline & \multicolumn{4}{c}{$\begin{array}{c}\text { Puppy affected with swimming } \\
\text { puppy syndrome }\end{array}$} \\
\cline { 2 - 5 } Parameter & 1 & 2 & 3 & 4 \\
\hline Sex & Male & Male & Female & Female \\
Age (weeks) & 8 & 8 & 9 & 8 \\
Weight (kg) & 2.5 & 2.7 & 2.5 & 2.2 \\
$\begin{array}{c}\text { Number of } \\
\text { puppies/litter }\end{array}$ & 5 & 4 & 5 & 5 \\
\hline
\end{tabular}

Table 3. Comparison of complete blood counts and blood chemistry between puppies with swimming puppy syndrome and healthy puppies.

\begin{tabular}{|c|c|c|c|c|c|c|c|}
\hline & \multirow{2}{*}{$\begin{array}{l}\text { Normal } \\
\text { range }^{\dagger}\end{array}$} & \multicolumn{2}{|c|}{ First visit } & \multirow{2}{*}{$P$-value } & \multicolumn{2}{|c|}{ After 1 month } & \multirow{2}{*}{$P$-value } \\
\hline & & Affected & Healthy & & Affected & Healthy & \\
\hline Hematocrit (\%) & $29-34^{\ddagger}$ & $33.25 \pm 4.19$ & $35.75 \pm 5.56$ & 0.306 & $35.50 \pm 3.79$ & $37.25 \pm 5.56$ & 0.357 \\
\hline Hemoglobin (g/dl) & $9.0-11.9^{\ddagger}$ & $10.08 \pm 0.65$ & $10.20 \pm 0.59$ & 0.390 & $10.58 \pm 1.15$ & $10.30 \pm 0.48$ & 0.357 \\
\hline $\mathrm{RBC}\left(10^{12} / \mathrm{L}\right)$ & $4.0-5.1^{\ddagger}$ & $4.75 \pm 0.42$ & $4.95 \pm 0.44$ & 0.278 & $5.10 \pm 0.29$ & $5.45 \pm 0.25$ & 0.097 \\
\hline WBC count $\left(10^{9} / \mathrm{L}\right)$ & $9.80-26.70^{\ddagger}$ & $26.50 \pm 5.20$ & $22.00 \pm 3.92$ & 0.186 & $23.00 \pm 4.24$ & $24.50 \pm 5.80$ & 0.374 \\
\hline Platelets $\left(10^{9} / \mathrm{L}\right)$ & $132-573^{\ddagger}$ & $303.5 \pm 91.91$ & $360.50 \pm 73.44$ & 0.260 & $323.50 \pm 103.67$ & $373.50 \pm 52.05$ & 0.280 \\
\hline AST (U/L) & $7-19^{\dagger}$ & $15.00 \pm 5.72$ & $13.25 \pm 7.09$ & 0.390 & $16.75 \pm 8.06$ & $14.50 \pm 4.65$ & 0.318 \\
\hline ALT (U/L) & $\leq 29^{\dagger}$ & $26.75 \pm 12.58$ & $19.50 \pm 8.10$ & 0.079 & $29.75 \pm 15.82$ & $20.25 \pm 6.40$ & 0.072 \\
\hline ALP (U/L) & $88-532^{\dagger}$ & $517.00 \pm 169.02$ & $498.00 \pm 112.33$ & 0.407 & $472.25 \pm 105.30$ & $440.25 \pm 74.76$ & 0.185 \\
\hline BUN (mg/dl) & $9.8-37.3^{\dagger}$ & $26.50 \pm 6.45$ & $25.50 \pm 10.340$ & 0.445 & $24.50 \pm 4.04$ & $26.00 \pm 8.16$ & 0.392 \\
\hline Creatinine (mg/dl) & $0.39-0.49^{\dagger}$ & $0.55 \pm 0.29$ & $0.53 \pm 0.33$ & 0.404 & $0.50 \pm 0.26$ & $0.53 \pm 0.19$ & 0.394 \\
\hline CK (U/L) & $31-255^{\dagger}$ & $563.00 \pm 80.55$ & $233.50 \pm 26.66$ & $0.003^{*}$ & $428.75 \pm 74.75^{\#}$ & $214.00 \pm 25.66$ & $0.008^{*}$ \\
\hline
\end{tabular}

Cited from Rosset et al., 2012 [6]; ${ }^{\dagger}$ Cited from Gorman, 2011 [7]; ${ }^{*}$ significant difference $(P<0.05)$ between affected and healthy groups; ${ }^{\#}$ significant difference $(P<0.05)$ between first visit and 1 month later in the same group. AST: aspartate aminotransferase; ALP: alkaline phosphatase; ALT: alanine aminotransferase; BUN: blood urea nitrogen; CK: creatine kinase. Data are expressed as mean $\pm \mathrm{SD}$. 


\section{Discussion}

The complete blood counts and blood chemistry profiles of affected puppies and healthy puppies were compared (Table 3). The results showed a significantly higher level $(P<0.05)$ of serum $\mathrm{CK}$ in animals with swimming puppy syndrome $(563.00 \pm 80.55 \mathrm{U} / \mathrm{L})$ compared with healthy puppies $(233.50 \pm 26.66 \mathrm{U} / \mathrm{L})$; moreover, the means were higher than the normal range $(31-255 \mathrm{U} / \mathrm{L})$. The other blood parameters showed no significant differences between the two groups, and were within the normal range. After 1 month, serum CK levels in the swimming puppy syndrome group $(428.75 \pm 74.75 \mathrm{U} / \mathrm{L})$ had significantly decreased $(P<0.05)$; however, compared to the healthy puppy group this value was still significantly higher $(P<0.05)$.

Creatine kinase is mostly present in the skeletal muscle, myocardium, brain and intestine. Sex has no influence on plasma CK activity, but it is higher in young dogs than adults $[2,3]$. Clinically, CK is widely used as a diagnostic tool for detection of muscular disorders such as muscular dystrophy $[4,5]$. Abnormal elevation of CK can result in muscle damage or injury, as well as leakage of the enzyme from the cytoplasm of myocytes. However, muscle damage typically occurs when CK levels are at least 100 times greater than normal. In the present study the elevation of serum CK in affected puppies was only double the normal value. It is possible that swimming puppy syndrome affects the metabolism of muscles, resulting in muscle hypoplasia or atrophy, but it does not cause severe muscle damage. An important limitation of this study was that biopsies of the affected muscles to evaluate muscle fiber were not performed, due to ethical considerations and because dog owners did not give the veterinarian permission to do this.

The results of this study show that complete blood counts and the levels of most blood chemicals are not useful for diagnosis of swimming puppy syndrome. Although serum CK was elevated in animals with swimming puppy syndrome compared with control puppies and with normal levels, it cannot be used for diagnosis of this disease due to silent elevation. However, CK could be used as a tool for prognosis of the disease and to evaluate the efficacy of treatment.

\section{Acknowledgements}

The authors would like to acknowledge the National Research University Project under Thailand's Office of the Higher Education Commission, and Metta Pet Hospital, Chiang Mai, Thailand for their kind help.

\section{REFERENCES}

[1] C. Yardimci, A. Ozak, H. O. Nisbet and Y. S. Sirin, "Swimming Syndrome in Two Labrador Puppies," Kafkas Universitesi Veteriner Fakultesi Dergisi, Vol. 15, 2009, pp. 637-640.

[2] D. Boettcher, R. Schmidt, C. Rehfeldt, G. Thaller, H. H. Swalve and S. Maak, "Evaluation of MAFbx Expression as a Marker for Congenital Splay Leg in Piglets," Developments in Biologicals (Basel), Vol. 132, 2008, pp. 301306. doi:10.1159/000317175

[3] M. Aktas, D. Auguste, H. P. Lefebvre, P. L. Toutain and J. P. Braun, "Creatine Kinase in the Dog: A Review," Veterinary Research Communications, Vol. 17, No. 5, 1993, pp. 353-369. doi:10.1007/BF01839386

[4] J. Yasuda and K. Too, "Studies on Serum Creatine Phosphokinase Isoenzyme: Seven Cases of Tetraplegia in the Dog," Japanese Journal of Veterinary Research, Vol. 31, No. 3-4, 1983, pp. 115-123.

[5] J. E. Sykes, J. P. Dubey, L. L. Lindsay, P. Prato, M. R. Lappin, L. T. Guo, A. P. Mizisin and G. D. Shelton, "Severe Myositis Associated with Sarcocystis spp. Infection in 2 Dogs," Journal of Veterinary Internal Medicine, Vol. 25, No. 6, 2011, pp. 1277-1283. doi:10.1111/j.1939-1676.2011.00828.x

[6] E. Rosset, B. Rannou, G. Casseleux, K. Chalvet-Monfray and S. Buff, "Age-Related Changes in Biochemical and Hematologic Variables in Borzoi and Beagle Puppies from Birth to 8 Weeks," Veterinary Clinical Pathology, Vol. 41, No. 2, 2012, pp. 272-282. doi:10.1111/j.1939-165X.2012.00415.x

[7] M. E. Gorman, "Clinical Chemistry of the Puppy and Kitten," In: M. E. Peterson and M. A. Kutzler, Eds., Small Animal Pediatrics, Elsevier Saunders, St. Louis, 2012, pp. 259-275. 